\title{
COMPARISON OF TWO ONE-EQUATION SUBGRID MODELS IN RECIRCULATING FLOWS
}

\author{
SINIŠA KRAJNOVIĆ (sinisaetfd.chalmers.se) \\ Dept. of Thermo and Fluid Dynamics, Chalmers University of Tech- \\ nology, SE-41296 Göteborg, Sweden \\ DIRK MÜLLER* (dm@wuek.rwth-aachen.de) \\ Lehrstuhl für Wärmeübertragung und Klimatechnik, RWTH-Aachen, \\ Germany \\ LARS DAVIDSON (ladaetfd.chalmers.se) \\ Dept. of Thermo and Fluid Dynamics, Chalmers University of Tech- \\ nology, SE-41296 Göteborg, Sweden
}

\begin{abstract}
The present paper presents a comparison of two one-equation models in recirculating flows. Special attention is given to their ability to predict the reverse transport of turbulent energy ('backscatter'). This work is part of the project "Large Eddy Simulation for Computing the Flow Around Vehicles" (http ://www.tfd.chalmers.se/ lada/projects/sinisa/proright.html) in collaboration between the Department of Thermo and Fluid Dynamics at Chalmers and the CFD group at Volvo Car Corporation.
\end{abstract}

\section{Introduction}

The dynamic model proposed by Germano [4] has numerical stability problems. These problems are caused by negative values and a large fluctuation in the dynamic coefficient, $C$. To ensure numerical stability, the dynamic coefficient must be averaged in some homogeneous direction or be clipped in an ad hoc manner. The averaging is not applicable to three-dimensional flows, where there are no homogeneous directions. Furthermore, ad hoc modification should be avoided if we wish to develop 'universal' turbulence models. In his attempt to improve the dynamic model, Ghosal et al [5] attempted to optimize the equation for C globally, but still with the constraint that $C>0$. This optimization gave Fredholm's integral equation of the second kind, which is very expensive to solve.

\footnotetext{
* This work was carried out during the author's stay at Chalmers.
} 


\section{Subgrid models and their properties}

Two one-equation subgrid models are used in the present study. The first model was developed by Davidson [1] (OEM). This model has also successfully been applied to fully developed channel flow [9] and vortex shedding flow around square cylinders [16]. The modeled transport equation for the subgrid kinetic energy, $k_{s g s}$, reads

$$
\frac{\partial k_{s g s}}{\partial t}+\left(-y k_{s g s}\right)_{, j}=\left(\left(\langle C\rangle_{x y z} \Delta k_{s g s}^{\frac{1}{2}}+v\right)\left(k_{s g s}\right)_{, j}\right)_{, j}+2 v_{s g s} \bar{S}_{i j} \bar{S}_{i j}-C_{*} \frac{k_{s g s}^{\frac{3}{2}}}{\Delta} .
$$

Here,

$$
C=-\frac{\mathcal{L}_{i j} M_{i j}}{2 M_{i j} M_{i j}} ; M_{i j}=\widehat{\Delta} K^{\frac{1}{2}} \widehat{\bar{S}}_{i j}-\Delta \overbrace{k_{s g s}^{\frac{1}{2}} \bar{S}_{i j}} ; K=\overparen{k_{s g s}}+\frac{1}{2} \mathcal{L}_{i i}
$$

where $\mathcal{L}_{i j}$ denotes the dynamic Leonard stresses and $K \equiv \frac{1}{2} T_{i i}$ is the subgrid kinetic energy on the test level. The coefficient, $C_{*}$, has the form

$$
C_{*}^{n+1}=(P_{K}-\overparen{P}_{k_{s g s}}+\frac{1}{\Delta} \overbrace{C_{*}^{n} k_{s g s}^{\frac{3}{2}}}) \frac{\overparen{\Delta}}{K^{\frac{3}{2}}}
$$

with

$$
P_{k_{s g s}}=-\tau_{i j}^{a}-\overline{l i}_{j} ; \tau_{i j}^{a} \equiv \mathcal{T}_{f}\left(u_{i}, u_{j}\right)=-2 C \Delta k_{s g s}^{\frac{1}{2}} \bar{S}_{i j} .
$$

To ensure numerical stability, a constant value of $C$ in space, $\left(\langle C\rangle_{x y z}\right)$, is used in the momentum equations, which is computed with the requirement that the production in the whole computational domain remains the same, i.e.

$$
\left\langle 2 C \Delta k_{s g s}^{\frac{1}{2}} \bar{S}_{i j} \bar{S}_{i j}\right\rangle_{x y z}=2\langle C\rangle_{x y z}\left\langle\Delta k_{s g s}^{\frac{1}{2}} \bar{S}_{i j} \bar{S}_{i j}\right\rangle_{x y z} .
$$

All local dynamic information is included through the source terms. This is physically more sound since large local variations in $C$ appear only in the source term and the effect of the large fluctuations in the dynamic coefficients will be smoothed out. The coefficients in the one-equation model affect the stresses in only an indirect way. In the standard dynamic model, the $C$ coefficient is linearly proportional to the stresses.

The second model studied in this paper is the localized dynamic $k_{s g s}$-equation model (LDKM) proposed by Menon and Kim [11]. In the LDKM, the following transport equation is solved:

$$
\frac{\partial k_{s g s}}{\partial t}+\left({ }^{-} y k_{s g s}\right)_{, j}=\left(\left(C \Delta k_{s g s}^{\frac{1}{2}}+v\right)\left(k_{s g s}\right)_{, j}\right)_{, j}+2 v_{s g s} \bar{S}_{i j} \bar{S}_{i j}-C_{*} \frac{k_{s g s}^{\frac{3}{2}}}{\Delta} .
$$


where

$$
C=\frac{1}{2} \frac{\mathcal{L}_{i j}^{a} \sigma_{i j}}{\sigma_{i j} \sigma_{i j}} ; \sigma_{i j}=-\widehat{\Delta} k_{t e s t}^{\frac{1}{2}} \overparen{\bar{S}_{i j}}
$$

and

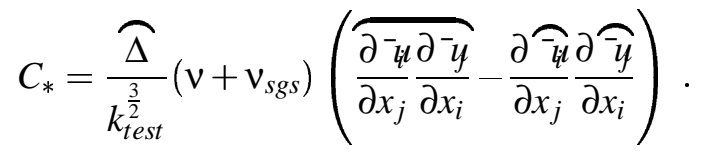

If we follow Vreman [17], SGS models should share some basic properties with the exact SGS stress tensor, $\tau_{i j}$.

i) Since $\tau_{i j}$ is a symmetric tensor, the modeled $\tau_{i j}$ should also be symmetric.

ii) The Navier - Stokes equations and their filtered form are Galilean invariant. They should retain this property even after $\tau_{i j}$ is replaced by the model.

iii) Since $\tau_{i j}$ should remain positive definite for positive filters, the model for $\tau_{i j}$ should remain positive definite as well if a positive filter is applied.

Both models fulfill symmetry of $\tau_{i j}$ and Galilean invariants. They also fulfill realizability conditions if following constraints are put on coefficient C: [8]

$$
\frac{k_{s g s}^{1 / 2}}{3 \Delta \bar{S}_{33}} \leq C \leq \frac{k_{s g s}^{1 / 2}}{3 \Delta \bar{S}_{11}}, \quad \bar{S}_{11} \geq \bar{S}_{22} \geq \bar{S}_{33}, \quad \bar{S}_{33} \leq 0
$$

and

$$
-\frac{2}{\sqrt{3}} \frac{k_{s g s}^{1 / 2}}{\Delta|\bar{S}|} \leq C \leq \frac{2}{\sqrt{3}} \frac{k_{s g s}^{1 / 2}}{\Delta|\bar{S}|}
$$

\section{Results}

This work uses a 3-D finite-volume method for solving the incompressible NavierStokes equations. Both convective and viscous plus subgrid fluxes are approximated by central differences of second-order accuracy. A Crank-Nicolson secondorder scheme was used for time integration. The momentum equations are solved with the Gauss-Seidel method whereas a multigrid V-cycle is used for the acceleration of convergence when solving the pressure equation $[2,3,7]$.

\section{Flow around a surface-mounted cube}

The first case selected for simulation in this work was the flow around a surfacemounted cubical obstacle placed on a channel wall at $R e=U_{b} H / v=40000$ based 
TABLE I. Mean and RMS values of lift and drag coeffi cients and lengths for reattachment and separation (see Fig. 5a).

\begin{tabular}{llllllll}
\hline Model & $\left\langle C_{D}\right\rangle_{t}$ & $C_{D, r m s}$ & $\left\langle C_{L}\right\rangle_{t}$ & $C_{L, r m s}$ & $X_{F 1}$ & $X_{T}$ & $X_{R 1}$ \\
\hline Exp. & - & - & - & - & 1.04 & - & 1.61 \\
OEM & 1.14 & 0.062 & 0.92 & 0.038 & 0.97 & 0.92 & 1.44 \\
LDKM & 1.16 & 0.070 & 0.91 & 0.040 & 1.06 & 0.92 & 1.38 \\
\hline
\end{tabular}

on the incoming mean bulk velocity, $U_{b}$, and the obstacle height, $\mathrm{H}$. The cube is located between $x=0$ and $x=1$ and the channel height is $h=2 H$. A computational domain with an upstream length of $x_{1} / H=3$ and a downstream length of $x_{2} / H=6$ was used, while the span-wise width was set to $b / H=7$. Even if the geometry of the flow configuration is rather simple, the flow is physically quite complex with multiple separation regions and vortices. A mesh of $82 \times 50 \times 66$ nodes was used. Near the walls of the cube $y_{\min }^{+}=3.7$, while on the top of the cube $y_{\min }^{+}=5.2$. The time step was set to 0.02 , which gave a maximum $C F L$ number of approximately 2 .

\section{BOUNDARY CONDITIONS}

The experimental profile (constant in time) was used at the inlet. The lateral boundaries were treated as slip surfaces. At the downstream boundary, a convective boundary condition $\frac{\partial u_{i}}{\partial t}+c \frac{\partial u_{i}}{\partial x}=0$ was used. Here, $c$ is the mean bulk velocity $U_{b}$. No-slip conditions were used at the upper and lower surfaces.

\section{GLOBAL QUANTITIES}

Both OEM [1] and LDKM [11] are used in the present study. The mean and RMS lift and drag coefficients are presented in Table I. The time history of $C_{D}$ and $C_{L}$ is given in Fig. 1a. There are no experimental values for drag and lift coefficients known to the authors. The values of mean and RMS values for OEM and LDKM are very similar.

\section{STATISTICS OF THE MEAN FLOW}

A series of time-averaged resolved velocities and turbulent stresses are computed and compared with the experiments. These results are presented in Fig. 2. As can be seen, the predictions without a model give poor agreement, whereas the two subgrid models give good agreement with experiments. The separation region at the top of the cube without a model is much too thin. This is probably because, without a model, the resolved fluctuations are not damped by any subgrid vis- 

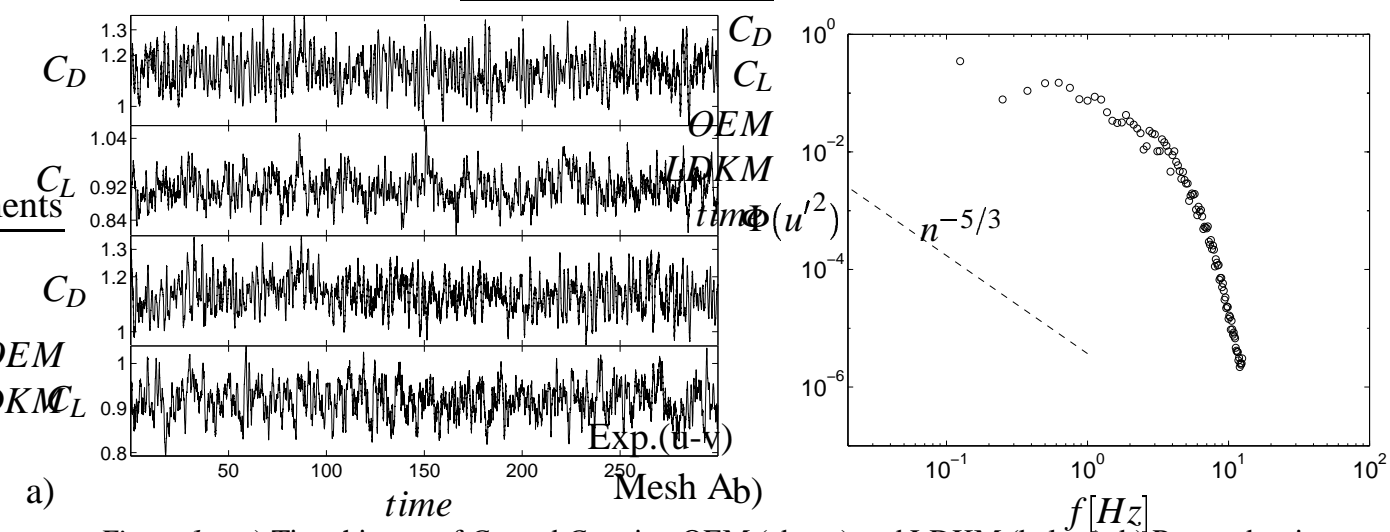

Figure 1. a) Time history of $C_{D}$ and $C_{L}$ using OEM (above) and LDKM (below). b) Power density spectrum at one chosen cell.

cosity, and the resolved fluctuations consequently become too large. This gives too large a turbulent diffusion, making the separation region smaller and thinner. It can be seen in Fig. 2 that the resolved shear stress $\left\langle u^{\prime} v^{\prime}\right\rangle_{t}$ without a model is not larger than those obtained with a model; however, care should be taken when comparing these, since the time-averaged velocity fields are very different. Instead, we could argue as follows: the resolved shear stress without a model is of the same magnitude as with a model, although the velocity gradient of the time-averaged velocity field without a model is much smaller; thus, taking into account the difference in the time averaged velocity fields, the resolved shear stress without a model is indeed larger. Both one-equation models gave similar results, but OEM gave perhaps slightly better results downstream in the wake region. The effect of the models is noticeable in a comparison with the calculation without a model. These differences are especially noticeable close to the roof of the cube and far downstream. The case studied in this paper was a test case at the 6th ERCOFTAC/IAHR/COST Workshop on Refined Flow Modelling in Delft (1997) using RANS Models. The velocity profiles, especially further downstream of the cube, are much better predicted by LES in the present work. The turbulence stresses are in significantly better agreement with the experimental values. Oscillations are present in the mean velocity profile $\left\langle{ }^{-} u\right\rangle$ for $x=-1.0$, as can be seen in Fig. 2. This is probably due to a combination of coarse mesh in that part of the domain and use of the central differencing scheme. In the case of shear stresses $u^{\prime} v^{\prime}$, both the resolved quantity $\left\langle u^{\prime} v^{\prime}\right\rangle_{t}$ and the SGS quantity $\left\langle\tau_{12}\right\rangle_{t}$ were computed as suggested by Reynolds [13]. We also computed $\left\langle u^{\prime 2}\right\rangle_{t}+\left\langle\tau_{11}\right\rangle_{t},\left\langle v^{\prime 2}\right\rangle_{t}+\left\langle\tau_{22}\right\rangle_{t}$ and $\left\langle w^{\prime 2}\right\rangle_{t}+\left\langle\tau_{33}\right\rangle_{t}$ and compared them with the experiments. Generally, the results for the velocities are in much better agreement with the experiment than the stresses. The difference between resolved mean turbulent shear stress and total (i.e. resolved plus SGS) turbulent shear stress is almost negligible. 
In Fig. 3, the oil-film visualization by Martinuzzi and Tropea [10] is compared with streamlines projected onto the floor. The predicted streamline pictures show most of the details observed in the experiments. In the experiments, Martinuzzi and Tropea observed three main curves in front the cube. Curve A corresponds to the primary, upstream separation curve and curve B corresponds to the approximate time-averaged location of the horseshoe vortex. Curve $\mathrm{C}$ indicates a secondary recirculation at the front base of the cube [10]. Curves A and $\mathrm{C}$ are very clearly visible in the picture of the predicted streamlines, while curve $B$ is somewhat weaker. The uncertainty of the experiment in this region is very large, and the flow between curves A and B is unstable. From this we conclude that it is not clear whether experiments or LES give better results in this part of the domain. The contour of the recirculation downstream of the cube is also clearly visible. Because of the inability to average over statistically equivalent points, the symmetry was used as a measure of whether the simulation was run for a sufficiently long time. The averaging time in the simulation was $150 H / U_{b}(3750$ time steps). As can be seen in Fig. 3, the surface streamlines downstream the cube are not fully symmetric, which indicates that the number of averaging samples is too small. Figure 4, plots the streamlines in the symmetry plane. The arch vortices and the head of the horseshoe are clearly visible in this picture. The re-attachment length, $X_{R 1}$, and separation lengths, $X_{F 1}$, and $X_{T}$ (Fig. 5a), are determined from the distribution of the skin friction coefficient $C_{f}=2 \tau_{w} / \rho U_{b}$ shown in Fig. 5b). Comparisons of different time-averaged recirculation lengths with experiments are shown in Table I.

\section{THE REVERSE FLOW OF ENERGY}

Special care was given to the phenomenon of 'backscatter'. It is well known that, in addition to the transport of the turbulent energy from large to small scales, the reverse transport is also possible ('backscatter'). Both one-equation models are able to predict negative production ('backscatter'). Depending on how large a fraction of the total energy transport is contained in the reverse transport, backscatter can be of importance. Constant $C$ in the model $v_{s g s}=C \Delta k_{s g s}^{1 / 2}$ is allowed to be negative in the production term in both OEM and LDKM. When $C$ becomes negative, it is believed that it represents 'backscatter'. Production term $P_{k_{s g s}}=-\tau_{i j}^{a}{ }^{-}{ }_{i, j}$ was studied both instantaneously and in a time-averaged way in Figs. 6b), 7 and 8. The LDKM gives a smaller magnitude of negative $P_{k_{s g s}}=-\tau_{i j}^{a}-\xi_{i j}$, than OEM. The strongest backscatter occurs near the front vertical corners, see Figs. $6 \mathrm{~b}, 7$ and 8 . The lower values of negative $P_{k_{s g s}}$ follow the horseshoe in the case of OEM, Fig. 7b). One iso-surface of the mean production term for LDKM is shown in Fig. 8a. It can be seen that the strongest backscatter is more uniformly distributed near the front vertical corners than in OEM. In Fig. 8b) it can be seen that LDKM predicts backscatter far upstream of the cube, in regions where the grid is refined. 

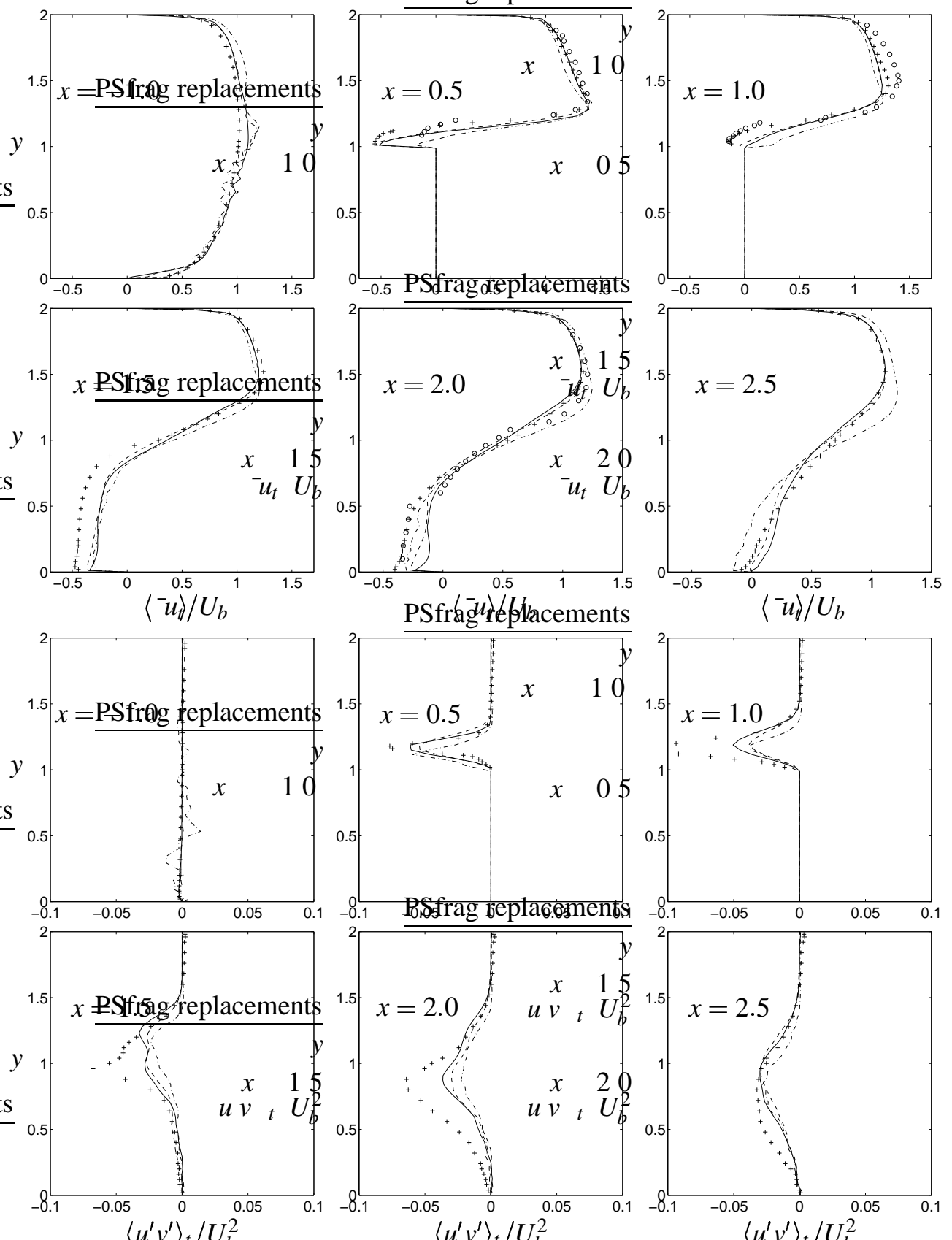

Figure 2. Surface-mounted cube. Comparison between OEM (dashed line), LDKM (solid line) and calculation without model (dash-dotted line). 
a)

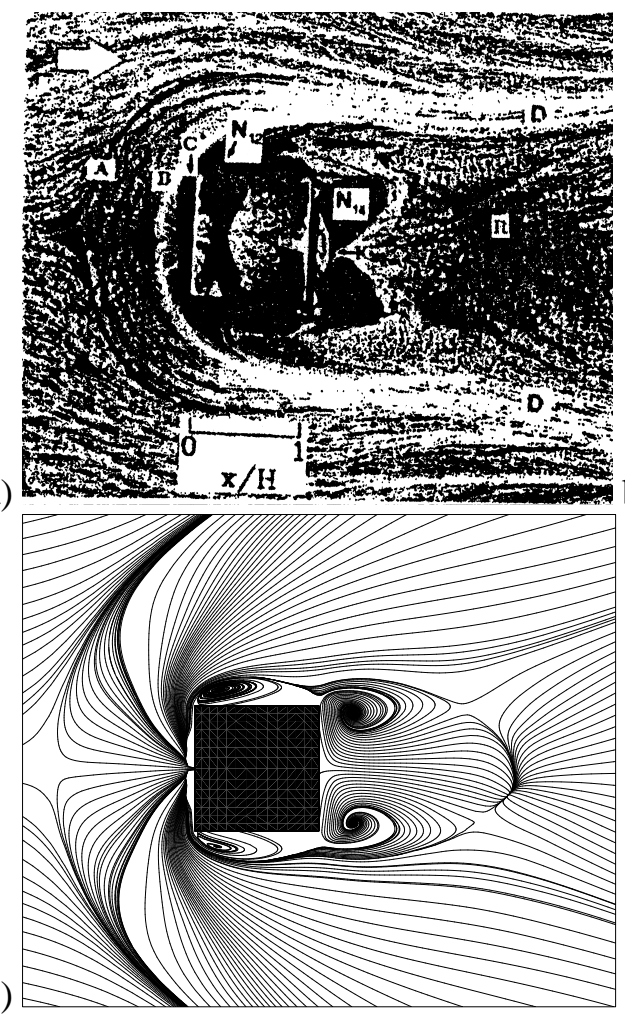

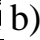

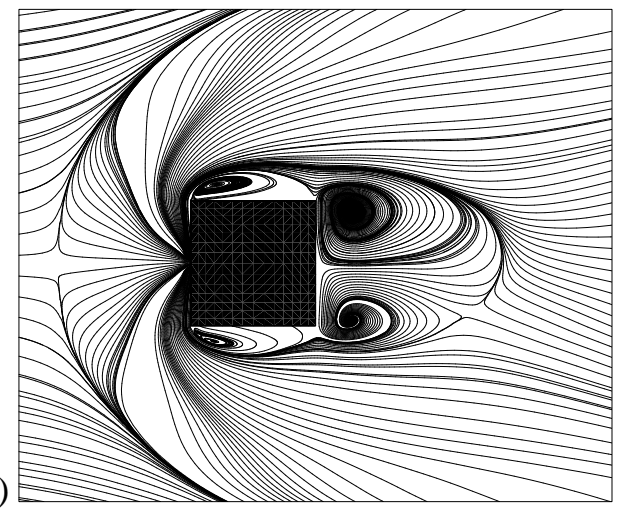

d)

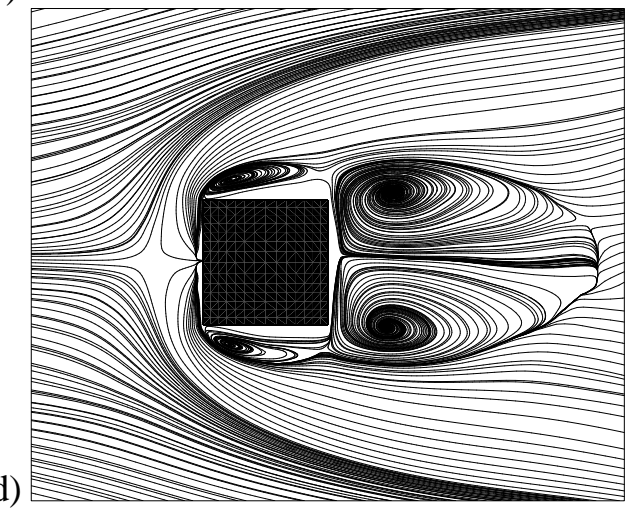

Figure 3. a) Oil-fi lm visualization by Martinuzzi and Tropea compared with streamlines of the mean flow projected onto the channel floor for LES with b) OEM, c) LDKM and d) without a model.

Thus LDKM seems to be more sensitive to grid refinement than OEM; this is because LDKM is more local than OEM. One can also find low-value backscatter located in the recirculation zone in front and on the roof of the cube, Fig. 8b).

\section{SENSITIVITY TO GRID REFINEMENT}

Sensitivity to grid refinement in both time and space was studied. A similar study using the Smagorinsky model is reported in [7]. It is very difficult to study sensitivity to grid refinement because refining the grid also changes the model. This is because $\Delta$ in $v_{s g s}=C \Delta k_{s g s}^{\frac{1}{2}}|\bar{S}|$ is defined as $\Delta=\left(\Delta_{1} \Delta_{2} \Delta_{3}\right)^{1 / 3}$. It is possible to define $\bar{\Delta}$ so that it is mesh independent, but this would drastically increase the cost of the calculation. We found that this mesh with only 270600 nodes gave results comparable with results from the LES workshops $[6,14]$, where some participants used more than $10^{6}$ nodes. In the present study, a refinement of the mesh did not produced obviously better results. The power density spectrum for the resolved streamwise fluctuation $u^{\prime 2}$ is shown in Fig. 1b. We can see that there is a tendency 

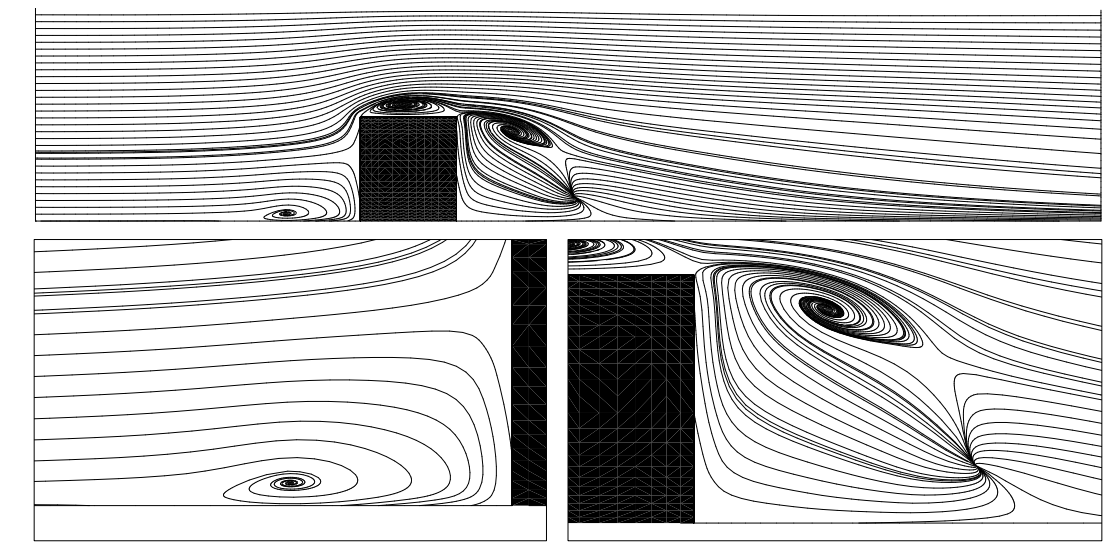
a)

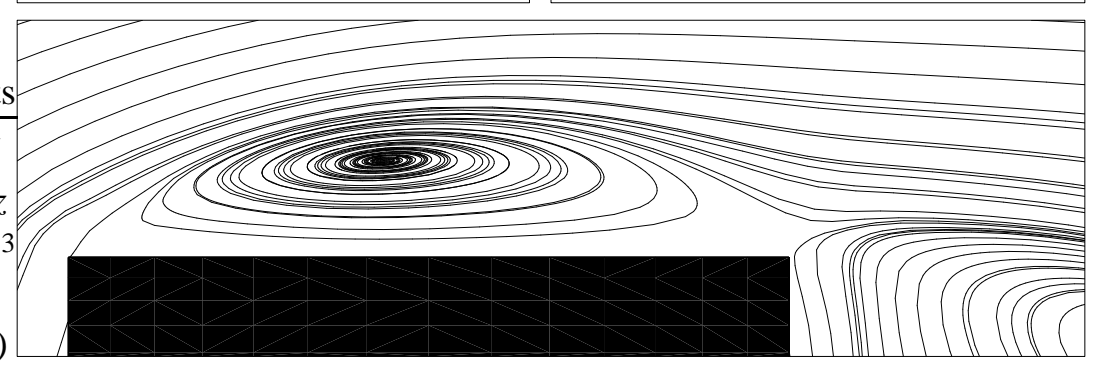

Figure 4. Streamlines of the mean flow projected onto the center-plane of the cube using LDKM.

a)

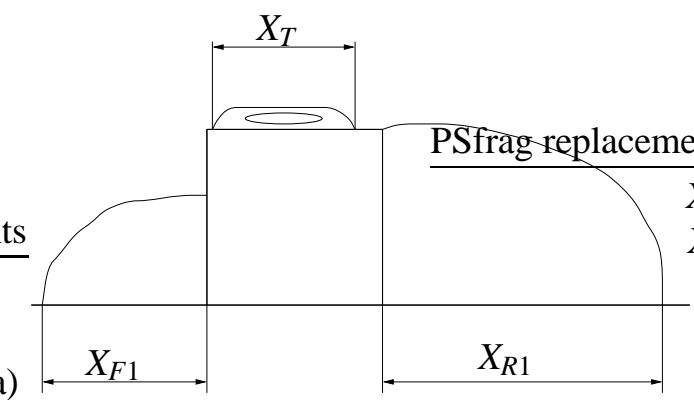

b)

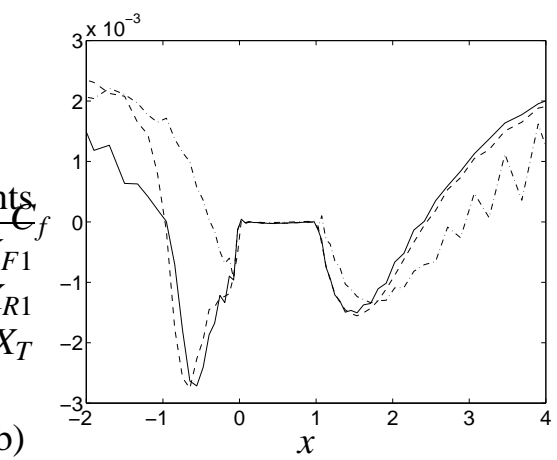

Figure 5. a) Re-attachment and separation lengths in the cube flow. b) Skin friction coeffi cient on the channel floor and roof of cube. Solid line: LDKM; dashed line: OEM; dash-dotted line: no model

to 'inertial region' $\left(\Phi \propto n^{(-5 / 3)}\right)$.

\section{NUMERICAL STABILITY}

The time history of the dynamic coefficient, $C$, and the dissipation coefficient, $C_{*}$, are shown for the two subgrid models in Fig. 6. It can be seen that the time history 

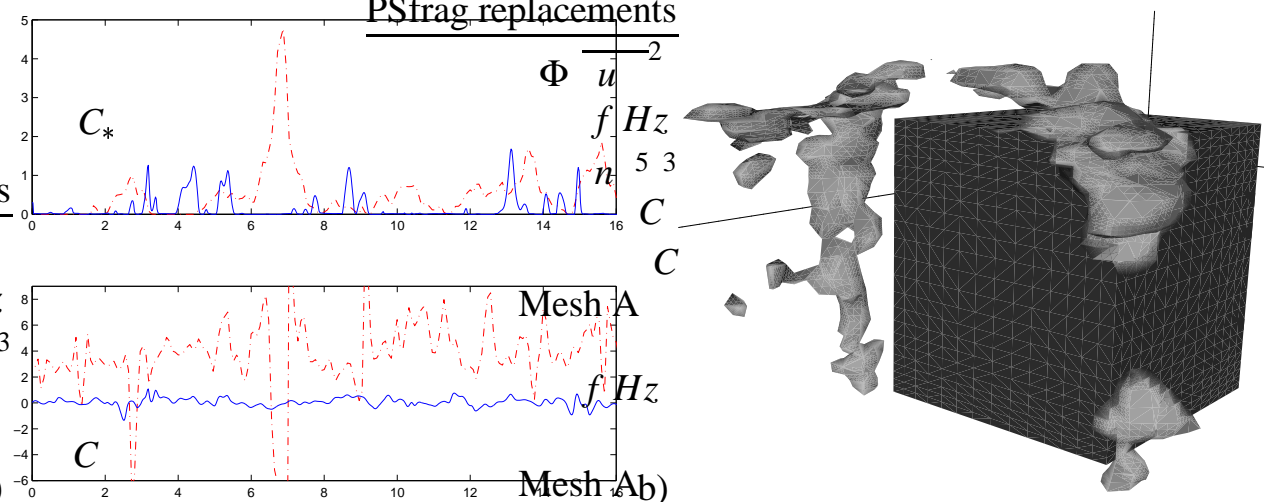

a)

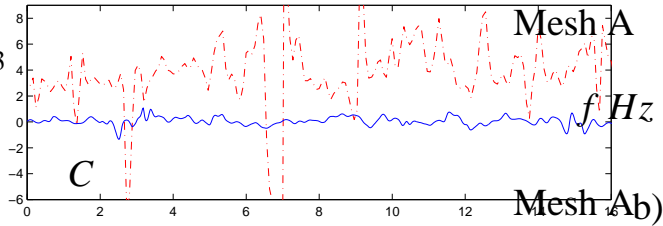

Figure 6. a) The dynamic coeffi cients $C_{\star}$ and $C$. Solid line: LDKM; dash-dotted line: OEM. b) The iso-surface of the instantaneous production term $P_{k_{s g s}}=-1$ in OEM

a)

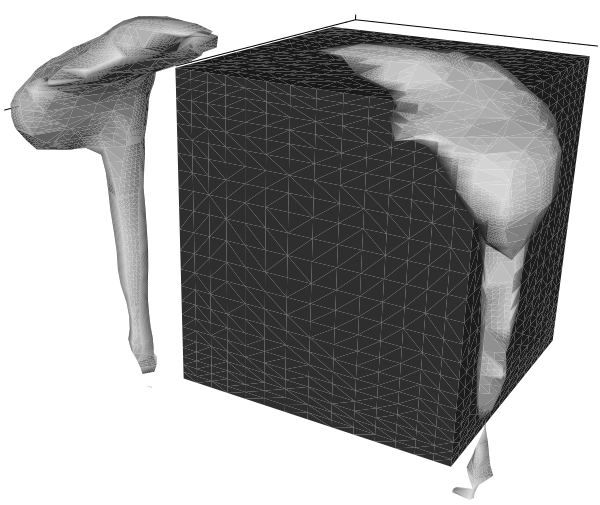

b)

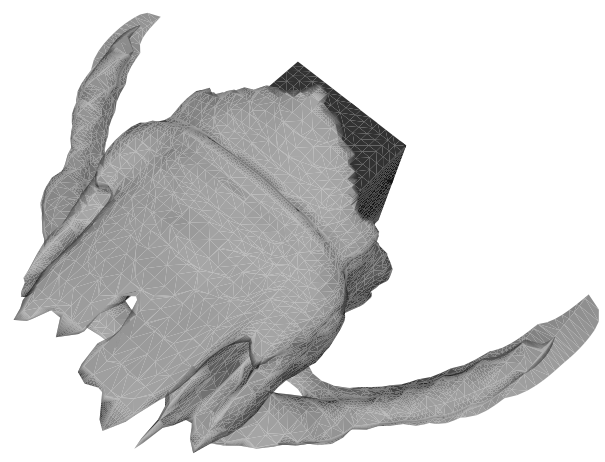

Figure 7. The iso-surface of the time-averaged production term in OEM. a) $\left\langle P_{k_{s g s}}\right\rangle_{t}=-1$, b) $\left\langle P_{k_{s g s}}\right\rangle_{t}=-0.05$

a)

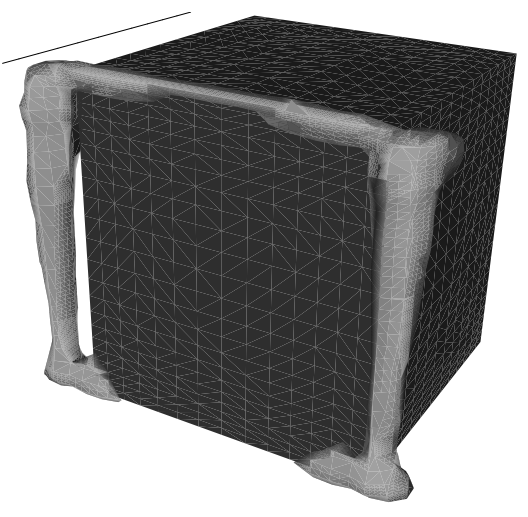

b)

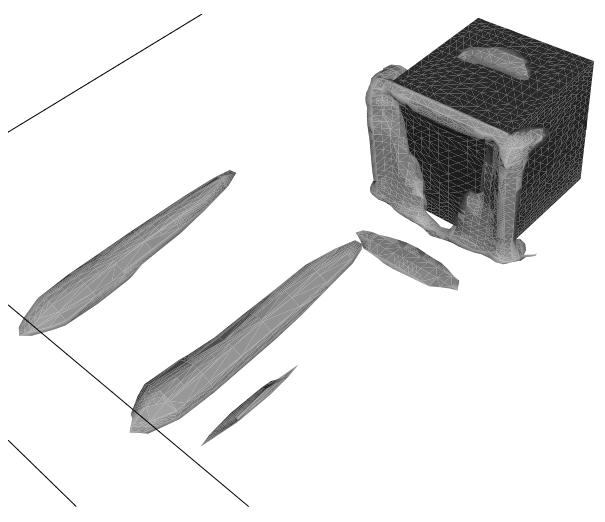

Figure 8. The iso-surface of the time-averaged production term in LDKM. a) $\left\langle P_{k_{s g s}}\right\rangle_{t}=-0.05$, b) $\left\langle P_{k_{s g s}}\right\rangle_{t}=-0.01$ 
of $C$ is much smoother for LDKM than for OEM. In OEM, similarity assumptions are made between the grid level (length scale $\ell<\Delta$, velocity scale $k_{s g s}^{\frac{1}{2}}$ ) and the test level (length scale $\ell<\widehat{\Delta}$, velocity scale $K^{\frac{1}{2}}$ ). However, in LDKM, the similarity assumption is made between the grid level and the intermediate level (length scale $\Delta<\ell<\widehat{\Delta}$, velocity scale $\left.\left[0.5 \mathcal{L}_{k k}\right]^{\frac{1}{2}}\right)$.

The denominator in Eq. 7, $\sigma_{i j} \sigma_{i j}$, does not tend to zero as much as the denominator, $M_{i j} M_{i j}$, in OEM (Eq. 2), which explains why $C$ does not oscillate as much in LDKM. In LDKM, the local coefficient is used in the momentum equation (with the restriction $v+v_{s g s} \geq 0$ ), which makes the model less stable. The time history of coefficient $C_{*}$ in front of the dissipation term (see Eqs. 3 and 8 ) is also smoother in LDKM than in OEM, although the difference is much smaller than for $C$. The dissipation coefficient often tends to zero (it is restricted so that $C_{*} \geq 0$ ) but never becomes larger than 5 .

\section{Conclusions}

A comparison of two one-equation subgrid models is made. Both models gave results in very good agreement with experiments. Results for statistics of the mean flow in computation with the two models were compared with computation without a model. The prediction with the two models gave better results then computation without a model. Poor agreement in the wake region is due to the coarse mesh in that part of the domain.

One-equation SGS models offer a number of advantages over the Germano model.

1. One-equation models can predict backscattering.

2. In the Germano model the dynamic coefficient must be clipped and/or averaged in the homogeneous direction(s). In one-equation models the local values of the dynamic coefficients can be used.

3. Although an additional transport equation need to be solved, one-equation models are often computationally cheaper than the Germano model, thanks to greater numerical stability [15].

4. The normal SGS stresses can be computed. This is important when predicting acoustics with LES [12].

\section{ACKNOWLEDGMENT}

This work was supported by NUTEK and the Volvo Car Corporation. 


\section{References}

1. L. Davidson. Large eddy simulation: A dynamic one-equation subgrid model for threedimensional recirculating flow. In 11th Int. Symp. on Turbulent Shear Flow, volume 3, pages 26.1-26.6, Grenoble, 1997.

2. L. Davidson. LES of recirculating flow without any homogeneous direction: A dynamic oneequation subgrid model. In 2nd Int. Symp. on Turbulence Heat and Mass Transfer, pages 481-490, Delft, 1997. Delft University Press.

3. P. Emvin. The Full Multigrid Method Applied to Turbulent Flow in Ventilated Enclosures Using Structured and Unstructured Grids. PhD thesis, Dept. of Thermo and Fluid Dynamics, Chalmers University of Technology, Gothenburg, 1997.

4. M. Germano, U. Piomelli, P. Moin, and W.H. Cabot. A dynamic subgrid-scale eddy viscosity model. Physics of Fluids A, 3:1760-1765, 1991.

5. S. Ghosal, T.S. Lund, P. Moin, and K. Akselvoll. A dynamic localization model for large-eddy simulation of turbulent flows. Journal of Fluid Mechanics, 286:229-255, 1995.

6. K. Hanjalić and S. Obi. ERCOFTAC/IAHR/COST workshop on refi ned flow modeling. Delft University of Technology, 1997.

7. S. Krajnović. Large-eddy simulation of the flow around a surface-mounted single cube in a channel. Rept. 98/7, Dept. of Thermo and Fluid Dynamics, Chalmers University of Technology, Gothenburg, 1998.

8. S. Krajnović. Properties of the dynamic one-equation subgrid model. Rept. 99/5, Dept. of Thermo and Fluid Dynamics, Chalmers University of Technology, Gothenburg, 1999.

9. S. Krajnović and L. Davidson. Large-eddy simulation of the flow around a surface-mounted cube using a dynamic one-equation subgrid model (to be presented). In The First International Symp. on Turbulence and Shear Flow Phenomena, Santa Barbara, Sept. 12-15, 1999.

10. R. Martinuzzi and C. Tropea. The flow around surface-mounted prismatic obstacles placed in a fully developed channel flow. ASME: Journal of Fluids Engineering, 115:85-91, 1993.

11. S. Menon and W.-W. Kim. High Reynolds number flow simulations using the localized dynamic subgrid-scale model. 34th Aerospace Sciences Meeting, AIAA Paper 96-0425, Reno, 1996.

12. U. Piomelli, C.L. Streett, and S. Sarkar. On the computation of sound by large-eddy simulations. Journal of Engineering Mathematics, 32:217-236, 1997.

13. W.C. Reynolds. The potential and limitations of direct and large eddy simulations. Lecture Notes in Physics, 357, 1989.

14. W. Rodi, J. H. Ferziger, M. Breuer, and M. Pourquié. Workshop on LES of flows past bluff bodies. Rotach-Egern, Germany, 1995.

15. A. Sohankar. Numerical Study of Laminar, Transitional and Turbulent Flow Past Rectangular Cylinders. PhD thesis, Dept. of Thermo and Fluid Dynamics, Chalmers University of Technology, Gothenburg, 1998.

16. A. Sohankar, L. Davidson, and C. Norberg. A dynamic one-equation model for simulation of flow around a square cylinder. In W. Rodi and D. Laurence, editors, Engineering Turbulence Modelling and Experiments 4, pages 227-236. Elsevier, 1999.

17. B. Vreman, B. Geurts, and H. Kuerten. Realizability conditions for the turbulent stress tensor in large-eddy simulation. Physics of Fluids A, 6:4057-4059, 1994. 